\title{
Predictor-feedback synthesis in coordinate-free formation control under time-varying delays
}

\author{
Antonio Gonzalez, Rosario Aragues, Gonzalo Lopez-Nicolas, Carlos Sagues ${ }^{\text {a }}$

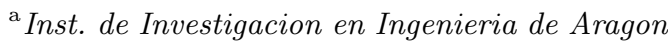 \\ Universidad de Zaragoza, C/Maria de Luna 1, E-50018 Zaragoza (Spain).
}

\begin{abstract}
This paper investigates new coordinate-free formation control strategies of multi-agent systems to overcome the negative effects of time delays. To this end, we present a single predictor-feedback scheme to compensate the multiple communication delays, assumed to be unknown but bounded and arbitrarily-fast time-varying. Although delays cannot exactly be compensated due to time-varying delay mismatches, the trade-off between robustness and convergence speed can be notably improved if the control gain is suitably designed. Hence, with the objective of enlarging the time-varying delay interval for a given convergence speed, an LMI-based iterative algorithm is presented to solve the control gain synthesis.
\end{abstract}

Key words: Multiagent systems, Formation stabilization, Robust control theory, Time-varying delay, Linear Matrix Inequality (LMI)

\section{Introduction}

In recent years, formation control for groups of autonomous mobile agents has received an increasing interest in the control community due to its broad potential applications in a large variety of areas, such as unmanned aerial vehicle (UAV) formation [1], search and rescue missions [2], cooperative transport [3], among others. One of the key aspects in these works is how to design a distributed control strategy based on local information exchange to achieve a geometrical formation shape in a coordinated fashion [4]. Formation control synthesis has been investigated under several strategies, including distance-based formation [5] and position-based formation in terms of absolute [6] and relative positions [7-9]. It is also interesting to consider that the agents' measurements frames are not equally

Email addresses: angonsor@unizar.es (Antonio Gonzalez), raragues@unizar.es (Rosario Aragues), gonlopez@unizar.es (Gonzalo Lopez-Nicolas), csagues@unizar.es (Carlos Sagues).

1 The authors would like to thank the reviewers for their helpful comments and suggestions.

2 This work was supported by projects PGC2018-098719B-I00 (MCIU/AEI/FEDER,UE), DGA T45-17R (Gobierno de Aragón), COMMANDIA SOE2/P1/F0638 (Interreg Sudoe Programme,ERDF) and Fundación Universitaria Antonio Gargallo (project 2018/B004). oriented. In this case, the gradient-descent formation control in local frames given in [9] has the advantage of achieving that the trajectories followed by the agents consist of straight line paths and globally converge to a unique rigid shape without a global coordinate system and leader agents, which brings more robustness and flexibility. The misalignment between orientation frames is addressed by introducing in the cost function to be minimized a rotation angle, on which the agents implicitly agree, capturing the method's independence of any global reference. Nevertheless, the unavoidable presence of delays may degrade the control-loop performance or even lead to instability [10,11], imposing constraints on the maximum achievable convergence speed by limiting the maximum control gain. Indeed, multi-agent systems constitute a networked system, where the available information is often delayed due to the time elapsed during data transmission between agents. Hence, time delays become a relevant issue in the stability analysis of formation control systems. Although the stability analysis in the presence of delays has been investigated in different problems involving cooperative control of multi-agent systems, such as consensus control $[12,13]$, leader-following formation control [14-16] and coordinate-free formation control [9], little attention has been paid to investigate advanced control strategies based on delay compensation [17-20] in order to counteract their negative effects. 
In [9], the coordinate-free formation control does not include delay compensation, and considers time-constant delays. Under these premises, the worst-case delay is expressed as a function of system parameters such as the number of agents or the decay rate. More recently, the similar problem was investigated in [21] but using multiple Smith predictors. Nevertheless, to the best authors' knowledge, two important limitations still remained open: (i) how to address the control gain synthesis in order to enlarge to the greatest extent the time-varying delay interval for a given convergence speed, and (ii) how to avoid the use of multiple delay compensators, which are required to exactly counteract each of the different delays but at the expense of increasing the complexity of the formation control for large number of agents.

The goal of this paper is to overcome the aforementioned limitations by integrating a novel predictor-feedback scheme in the coordinate-free formation control [9] assuming unknown but bounded time-varying delays. In particular, we introduce the following contributions: (i) a systematic algorithm to solve the control gain synthesis with the objective of enlarging the time-varying delay interval for a given convergence speed, and (ii) a single predictor-feedback delay compensator to counteract multiple time-varying delays, that is to say, without resorting to multiple delay compensators to deal with each delayed measurement. Moreover, we analyze the existing trade-offs between the maximum allowable delay interval and other system features, such as decay rate or the number of agents. The stability analysis and control synthesis approaches are based on Linear Matrix Inequalities (LMIs), which can efficiently be solved by means of convex optimization tools commercially available in software libraries (Matlab LMI toolbox [22], SEDUMI [23]).

\section{Problem statement and preliminaries}

Consider a multi-agent system formed by $N$ agents having single integrator kinematics,

$$
\dot{q}_{i}=u_{i}, \quad i=1, \ldots, N
$$

where $q_{i}^{T}=\left[q_{i}^{x}, q_{i}^{y}\right]$ is the $2-D$ position vector of each agent, referred to any arbitrary reference frame. For each pair of agents $j, i \in\{1, \ldots, N\} \times\{1, \ldots, N\}, j \neq i$, we define $q_{j i}=q_{j}-q_{i}$ and $c_{j i}$ respectively as the current and the desired relative position between them. The control action $u_{i}$ must be designed to force (1) to reach a prescribed target formation, defined by the following set of relative position vectors:

$$
c_{j i}^{T}=\left(c_{j i}^{x}, c_{j i}^{y}\right), \forall[j, i] \in\{1, \ldots, N\} \times\{1, \ldots, N\} .
$$

The following assumptions on the multi-agent system (1) and the coordinate-free formation control define the particular problem addressed regarding the communication topology, the reference system available, and the nature of communication delays $[9,24]$ :

Assumption 1 The inequality $\left|\sum_{j} \sum_{i} q_{j i}^{T} c_{j i}\right| \geq$ $H, \forall t \geq 0$ holds for some $H \in \mathcal{R}>0$. This assumption implies that initially not all the agents are close together and that they remain sufficiently separated throughout the control execution.

Assumption 2 The agents do not share a global reference frame and only relative position measurements between the agents are available.

Assumption 3 No particular communication topology is assumed, provided that all relative position measurements between the agents in local coordinates are available to them by means of multi-hop communication.

Assumption 4 All relative position measurements may be affected by unknown time-varying delays: $0 \leq \underline{\tau} \leq$ $\tau_{j i}(t) \leq \bar{\tau}, \forall(i, j) \in(1, \ldots, N) \times(1, \ldots, N), i \neq j$, where $\delta=\bar{\tau}-\underline{\tau}$ is the worst-case delay interval. Moreover, delays are not necessarily symmetric, i.e $\tau_{j i}(t) \neq \tau_{i j}(t)$, and arbitrarily-fast time-varying (i.e. not necessarily continuously differentiable functions).

Remark 1 Note that Assumption 3 does not imply that all the communication links are always active during control execution. In other words, if the communication link between two agents fails, the relative position between them can be transmitted via multi-hop communication protocols through one or multiple different communication links involving other neighbor agents. Thus, the time-varying delays that appear in the distributed propagation of the relative position measurements may be subject to abrupt changes (see Assumption 4).

\subsection{Preliminary results}

This section provides some helpful definitions and results:

Definition 1 [25] Given the system $M$ with input/output $\eta(t)$ and $y(t)$ respectively, the $H_{\infty}$ norm of system $M$ represents the largest possible $\mathcal{L}_{2}$-gain, provided by the system under zero initial conditions, i.e.,

$$
\|M\|_{\infty}=\sup _{\|\eta\|_{2} \neq 0} \frac{\|y\|_{2}}{\|\eta\|_{2}}, \quad \forall \eta \in \mathcal{L}_{2},
$$

where $\mathcal{L}_{2}=\left\{\eta(t): \int_{0}^{\infty}\|\eta(s)\|^{2} d s<\infty\right\}$ denotes the set of square integrable signals, and $\|\eta\|_{2}$ stands for the $\mathcal{L}_{2}$ norm of the input signal $\eta(t)$, defined as $\|\eta\|_{2}=$ $\sqrt{\int_{0}^{\infty}\|\eta(s)\|^{2} d s}$. 
Theorem 1 (Scaled Small Gain Theorem)[11] Given a known system $M$ and some unknown time-varying operator $\Delta$ with $\|\Delta\|_{\infty} \leq 1$, the interconnected system: $y=M \eta, \eta=\Delta y$ is robustly stable if the following two conditions hold: (i) The system $M$ is internally stable and (ii) there exist nonsingular matrices $\mathcal{T}_{1}, \mathcal{T}_{2}$ such that $\mathcal{T}_{1} \Delta=\Delta \mathcal{T}_{2}$ and $\left\|\mathcal{T}_{2} \circ M \circ \mathcal{T}_{1}^{-1}\right\|_{\infty}<1$

Lemma 1 [26, Lemma 2] Given any arbitrary signal $q(t)$, let $y_{d}(t)=\dot{q}(t)$ and

$$
\omega(t)=\frac{2}{\delta}\left(q(t-\tau(t))-\frac{1}{2}(q(t-\underline{\tau})+q(t-\bar{\tau}))\right),
$$

where $\tau(t)$ is a time-varying delay satisfying $0 \leq \underline{\tau} \leq$ $\tau(t) \leq \bar{\tau}$ with $\delta=\bar{\tau}-\underline{\tau}$. Then, the time-varying operator $\Delta_{d}: y_{d} \rightarrow \omega$ renders $\Delta_{d}=\frac{1}{\delta} \int_{-\tau}^{-\bar{\tau}} \kappa(\theta) y_{d}(t+\theta) d \theta$, where $\kappa(\theta)=1$ if $\theta \leq-\tau(t)$, and $\kappa(\theta)=-1$ otherwise. Moreover, the operator $\Delta_{d}$ satisfies $\left\|\Delta_{d}\right\|_{\infty} \leq 1$.

\section{$3 \quad$ Predictor-feedback formation control}

Let $e_{j i}$ be the relative position error between two agents $i$ and $j$ given by

$$
e_{j i}=q_{j i}-R\left(\alpha_{i}\right) c_{j i}
$$

where the relative position measurements $q_{j i}$ are expressed in each local agents' frame, and $R\left(\alpha_{i}\right) c_{j i}$ describes the rotated reference frame to be reached, where

$$
R\left(\alpha_{i}\right)=\left[\begin{array}{cc}
\cos \left(\alpha_{i}\right) & -\sin \left(\alpha_{i}\right) \\
\sin \left(\alpha_{i}\right) & \cos \left(\alpha_{i}\right)
\end{array}\right],
$$

and $\alpha_{i}$ must be agreed by all the agents using the locally available measurements $q_{j i}$.

For the multi-agent system (1) subject to time-varying communication delays $\underline{\tau} \leq \tau_{j i}(t) \leq \bar{\tau}$, we propose the formation control:

$$
u_{i}=K\left(\sum_{j} e_{j i}^{\tau}-\gamma \frac{N}{2} \int_{t-\underline{\tau}}^{t} u_{i}(s) d s-\gamma \frac{N}{2} \int_{t-\bar{\tau}}^{t} u_{i}(s) d s\right)
$$

where delay compensation is activated by setting $\gamma=1$. Note that, differently from the conventional Artstein's reduction method [27], we have considered two integral terms with $\underline{\tau}, \bar{\tau}$. Parameter $K$ is the control gain to be designed, and $\gamma=\{0,1\}$. The relative position errors $e_{j i}^{\tau}$ obtained from the available delayed measurements render

$$
e_{j i}^{\tau}=q_{j i}\left(t-\tau_{j i}(t)\right)-R\left(\alpha_{i}^{\tau}\right) c_{j i},
$$

where

$$
\begin{array}{r}
\alpha_{i}^{\tau}=\operatorname{atan} 2\left(\sum_{k} \sum_{j}\left(q_{k i}^{\tau_{k i}}-q_{j i}^{\tau_{j i}}\right)^{T} c_{k j}^{\perp}\right. \\
\left.\sum_{k} \sum_{j}\left(q_{k i}^{\tau_{k i}}-q_{j i}^{\tau_{j i}}\right)^{T} c_{k j}\right)
\end{array}
$$

and $c_{k j}^{\perp T}=\left[c_{k j}^{y},-c_{k j}^{x}\right]$. The function atan $2: \mathcal{R}^{2} \rightarrow$ $(-\pi, \pi]$ is equivalent to a four-quadrant arctangent function $[28,29]$, defined as

$$
\operatorname{atan} 2(y, x)= \begin{cases}0 & (x, y)=(0,0) \\ f(x, y) & \text { otherwise }\end{cases}
$$

where $f(x, y)=\arctan (y / x)+(\pi / 2) \operatorname{sign}(y)(1-$ $\operatorname{sign}(x))$, and $\operatorname{sign}(a)=1$ if $a \geq 0, \operatorname{sign}(a)=-1$ otherwise.

Remark 2 Note that multiple time-varying delays $\tau_{j i}(t), j=1, \ldots, N, j \neq i$ are handled by a single predictor-feedback component in (6) with two integral terms involving the lower and upper bounds $\underline{\tau}, \bar{\tau}$ respectively. Hence, delays cannot be exactly compensated unless $\tau_{j i}(t) \equiv \tau, \forall i, j, i \neq j, \forall t \geq 0$ (as shown later in Corollary 1). Nevertheless, larger time-varying delay interval $\delta$ can be achieved by suitably designing the control gain K, improving therefore robustness. Next sections address the stability analysis and control design by means of robust control theory and LMIs.

\section{Robust stability analysis}

This section deals with the robust asymptotic stability analysis of the proposed formation control. To this end, we obtain an equivalent delay-free model description of the closed-loop multi-agent system in Section 4.1, useful to address the robust stability analysis in Section 4.2 by small gain theory (see Theorem 1).

\subsection{Delay-free model description}

Let $\bar{q}$ be the augmented vectors containing the relative positions $q_{j i}, \forall i, j / j \neq i$ with the following arrangement:

$$
\begin{aligned}
& \bar{q}^{T}=\left(q_{21}, q_{31}, q_{41}, \cdots, q_{N 1},\right. \\
& q_{12}, q_{32}, q_{42}, \cdots, q_{N, 2}, \\
& \cdots, \cdots, \cdots, \cdots, \\
& \left.q_{1 N}, q_{2 N}, q_{3 N}, \cdots, q_{N-1, N}\right) .
\end{aligned}
$$

Also, let

$$
\bar{e}^{T}=\left[e_{21}, \cdots \cdots, e_{N 1}, \cdots, e_{1 N}, \cdots, e_{N-1, N}\right]
$$


be the augmented vector $\bar{e}$ gathering all the terms $e_{j i}$ defined in (7) with the same arrangement as (9). From $\bar{q}$ and $\bar{e}$, the following lemma finds a closed-loop delay-free model consisting in two interconnected systems $(y=M \eta$ and $\eta=\Delta y$ ) where the forward system $M$ is LTI, and the uncertain feedback system $\Delta$ is a norm-bounded operator which contains all sources of time-varying delays.

Lemma 2 The multi-agent system (1) with control law (6) is equivalent to the following delay-free interconnected system model:

$$
\left[\begin{array}{c}
\dot{\bar{q}} \\
\hline y
\end{array}\right]=\underbrace{\left[\begin{array}{c|c}
K \mathcal{A}_{s} & K \mathcal{B}_{s} L_{\delta} \\
\hline L_{K} \mathcal{C}_{s} & L_{K} \mathcal{D}_{s} L_{\delta}
\end{array}\right]}_{M}\left[\begin{array}{c}
\bar{e} \\
\bar{\eta}
\end{array}\right], \quad \eta=\Delta y,
$$

where $\bar{q}, \bar{e}$ are defined in (9), (10), $\eta \in \mathcal{R}^{2 \bar{N}+2 N}, y \in$ $\mathcal{R}^{2 \bar{N}+N}$ with $\bar{N}=N(N-1)$ are input and output vectors, which are interconnected by the uncertain block-diagonal feedback system $\Delta$ satisfying $\|\Delta\|_{\infty} \leq 1$, and

$$
\begin{aligned}
& \delta=\bar{\tau}-\underline{\tau}, \\
& L_{K}=\left[\begin{array}{cc}
K I_{2 \bar{N}} & 0 \\
0 & I_{N}
\end{array}\right], L_{\delta}=\left[\begin{array}{cc}
\delta I_{2 \bar{N}} & 0 \\
0 & I_{2 N}
\end{array}\right], \\
& \mathcal{A}_{s}=-N \cdot I_{2 \bar{N}}, \quad \mathcal{B}_{s}=\left[\begin{array}{cc}
\frac{1}{2} B_{1} & B_{2}
\end{array}\right], \\
& \mathcal{C}_{s}=\left[\begin{array}{c}
-N \cdot I_{2 \bar{N}} \\
0_{N \times 2 \bar{N}}
\end{array}\right], \quad \mathcal{D}_{s}=\left[\begin{array}{cc}
\frac{1}{2} B_{1} & B_{2} \\
D & 0_{N \times 2 N}
\end{array}\right],
\end{aligned}
$$

where

$$
\begin{aligned}
& B_{1}=\left(\left(Q_{1}-Q_{2}\right) \otimes 1_{1 \times(N-1)}\right) \otimes I_{2} \\
& B_{2}=-\left(\left(Q_{1}-Q_{2}\right) \cdot \operatorname{diag}(\bar{h})\right) \otimes I_{2} \\
& D=\operatorname{diag}\left(\bar{c}^{T} \cdot\left(\mathcal{I}_{N}^{1} \otimes I_{2}\right)^{T}, \cdots, \bar{c}^{T} \cdot\left(\mathcal{I}_{N}^{N} \otimes I_{2}\right)^{T}\right) \\
& Q_{1}^{T}=\left[\left(\mathcal{I}_{N}^{1}\right)^{T}, \cdots,\left(\mathcal{I}_{N}^{N}\right)^{T}\right] \\
& Q_{2}=I_{N} \otimes 1_{(N-1) \times 1} \\
& \bar{c}^{T}=\left[\sum_{j} c_{1 j}^{\perp T}, \cdots, \sum_{j} c_{N j}^{\perp T}\right], \\
& \bar{h}^{T}=\left[h_{1} \cdots h_{N}\right], h_{1 \leq i \leq N}=\frac{1}{H}\left\|\sum_{k} c_{k i}\right\|
\end{aligned}
$$

being $I_{n}$ the $n \times n$ identity matrix, and $\mathcal{I}_{n}^{p}$ a matrix of dimensions $n-1 \times n$, constructed from the identity matrix $I_{n}$ by removing its $p_{t h}$ row.

Proof: Consider the formation control (6) with $\gamma=1$ for two agents agents $j$ and $i$,

$$
\begin{aligned}
& u_{j}=K \sum_{k} e_{k j}^{\tau}-\frac{N K}{2}\left(\int_{t-\underline{\tau}}^{t} u_{j}(s) d s+\int_{t-\bar{\tau}}^{t} u_{j}(s) d s\right), \\
& u_{i}=K \sum_{k} e_{k i}^{\tau}-\frac{N K}{2}\left(\int_{t-\underline{\tau}}^{t} u_{i}(s) d s+\int_{t-\bar{\tau}}^{t} u_{i}(s) d s\right) .
\end{aligned}
$$

Now, define the following Artstein's state transformations:

$$
\begin{aligned}
& z_{i}=\frac{1}{2}\left(\sum_{k} q_{k i}(t-\underline{\tau})+\sum_{k} q_{k i}(t-\bar{\tau})\right) \\
& -\frac{N}{2} \int_{t-\underline{\tau}}^{t} u_{i}(s) d s-\frac{N}{2} \int_{t-\bar{\tau}}^{t} u_{i}(s) d s, \\
& z_{j}=\frac{1}{2}\left(\sum_{k} q_{k j}(t-\underline{\tau})+\sum_{k} q_{k j}(t-\bar{\tau})\right) \\
& -\frac{N}{2} \int_{t-\underline{\tau}}^{t} u_{j}(s) d s-\frac{N}{2} \int_{t-\bar{\tau}}^{t} u_{j}(s) d s .
\end{aligned}
$$

Let $\delta=\bar{\tau}-\underline{\tau}$. Applying (15), we can reformulate (14) as

$$
\begin{aligned}
& u_{j}=K z_{j}+\frac{\delta K}{2} \sum_{k} \omega_{k j}-K R\left(\alpha_{j}\right) \sum_{k} c_{k j}-K h_{j} \rho_{j} \\
& u_{i}=K z_{i}+\frac{\delta K}{2} \sum_{k} \omega_{k i}-K R\left(\alpha_{i}\right) \sum_{k} c_{k i}-K h_{i} \rho_{i}
\end{aligned}
$$

where the following inputs $\omega_{k j}, \omega_{k i}, \rho_{j}$ and $\rho_{i}$, which embeds all sources of time-varying delay mismatches, are respectively defined as

$$
\begin{aligned}
& \omega_{k j}=\frac{2}{\delta}\left(q_{k j}\left(t-\tau_{k j}(t)\right)-\frac{1}{2} q_{k j}(t-\underline{\tau})-\frac{1}{2} q_{k j}(t-\bar{\tau})\right), \\
& \omega_{k i}=\frac{2}{\delta}\left(q_{k i}\left(t-\tau_{k i}(t)\right)-\frac{1}{2} q_{k i}(t-\underline{\tau})-\frac{1}{2} q_{k i}(t-\bar{\tau})\right),
\end{aligned}
$$

and

$$
\begin{aligned}
& \rho_{j}=\frac{1}{h_{j}}\left(R\left(\alpha_{j}^{\tau}\right)-R\left(\alpha_{j}\right)\right) \sum_{k} c_{k j}, \\
& \rho_{i}=\frac{1}{h_{i}}\left(R\left(\alpha_{i}^{\tau}\right)-R\left(\alpha_{i}\right)\right) \sum_{k} c_{k i},
\end{aligned}
$$

where the scalars $h_{j}, h_{i}$ are defined in (13). The idea is to design the output systems so as the interconnection between them and the above inputs $\omega_{k j}, \omega_{k i}, \rho_{j}, \rho_{i}$ are norm-bounded, leading to the uncertain feedback system $\Delta$ given in (11) 
Note from Lemma 1 and the definition of $\omega_{j i}$ in (17) that there exists an unitary norm-bounded time-varying operator $\Delta_{q, j i}$ satisfying

$$
\omega_{j i}=\Delta_{q, j i} \cdot \psi_{j i}, \quad \psi_{j i}=\dot{q}_{j i} .
$$

From Assumption 1 and applying trigonometry, it can be deduced following the same outline as [24, Lemma 4] that the $\mathcal{L}_{2}$-induced norm of the time-varying operator mapping the input $\rho_{i}$ in (18) and the output

$$
\xi_{i}=\frac{\delta}{2} \sum_{k} \sum_{j}\left(\omega_{k i}^{T} c_{k j}^{\perp}-\omega_{j i}^{T} c_{k j}^{\perp}\right)=\delta \sum_{k}\left(\sum_{j} c_{k j}^{\perp T} \omega_{k i}\right)
$$

is bounded by $1, \forall t \geq 0$. Hence, there exists a unitary norm-bounded time-varying operator $\Delta_{\rho, i}$ satisfying $\rho_{i}=\Delta_{\rho, i} \cdot \xi_{i}$. From the multiagent system (1) we have that $\dot{q}_{j i}=\dot{q}_{j}-\dot{q}_{i}=u_{j}-u_{i}$. Hence, from (16), we obtain

$$
\begin{gathered}
\dot{q}_{j i}=K\left(z_{j}-z_{i}\right)-K\left(R\left(\alpha_{j}\right) \sum_{k} c_{k j}-R\left(\alpha_{i}\right) \sum_{k} c_{k i}\right) \\
+\frac{\delta K}{2}\left(\sum_{k} \omega_{k j}-\sum_{k} \omega_{k i}\right)-K h_{j} \rho_{j}+K h_{i} \rho_{i} .
\end{gathered}
$$

As a result, the forward systems (21) are LTI with timeconstant delays, and the two pairs of input-outputs $\left(\omega_{j i}, \psi_{j i}\right)$ and $\left(\rho_{i}, \xi_{i}\right)$ embeds all sources of time-varying delay mismatches into uncertain feedback systems $\Delta_{q, j i}$, $\Delta_{\rho, i}$. From (15) we obtain

$$
\begin{aligned}
& z_{j}-z_{i}=\frac{1}{2}\left(\sum_{k} q_{k j}(t-\underline{\tau})+\sum_{k} q_{k j}(t-\bar{\tau})\right) \\
& -\frac{N}{2}\left(\int_{t-\underline{\tau}}^{t} u_{j}(s) d s-\int_{t-\underline{\tau}}^{t} u_{i}(s) d s\right) \\
& -\frac{1}{2}\left(\sum_{k} q_{k i}(t-\underline{\tau})+\sum_{k} q_{k i}(t-\bar{\tau})\right) \\
& -\frac{N}{2}\left(\int_{t-\bar{\tau}}^{t} u_{j}(s) d s-\int_{t-\bar{\tau}}^{t} u_{i}(s) d s\right) .
\end{aligned}
$$

From (1) it can be deduced that

$$
\begin{aligned}
& \int_{t-\underline{\tau}}^{t} u_{j}(s) d s-\int_{t-\underline{\tau}}^{t} u_{i}(s) d s \\
& =\int_{t-\underline{\tau}}^{t} \dot{q}_{j i}(s) d s=q_{j i}-q_{j i}(t-\underline{\tau}), \\
& \int_{t-\bar{\tau}}^{t} u_{j}(s) d s-\int_{t-\bar{\tau}}^{t} u_{i}(s) d s \\
& =\int_{t-\bar{\tau}}^{t} \dot{q}_{j i}(s) d s=q_{j i}-q_{j i}(t-\bar{\tau}) .
\end{aligned}
$$

Replacing (23) into (22), and taking into account that $\sum_{k} q_{k j}-\sum_{k} q_{k i}=-N q_{j i}$, the delayed terms in (22) are cancelled, obtaining the equivalences $z_{j}-z_{i}=-N q_{j i}$. Note that $\alpha_{j}$ and $\alpha_{i}$ are the rotation angle computed by agents $j$ and $i$ assuming no delays, leading to $\alpha_{j}=\alpha_{i} \equiv$ $\alpha$ (see Remark 2). Hence, applying $\sum_{k} c_{k j}-\sum_{k} c_{k i}=$ $-N c_{j i}$ and $z_{j}-z_{i}=-N q_{j i}$ the expression (21) becomes delay-free, leading to

$$
\begin{aligned}
& \dot{q}_{j i}=-N K\left(q_{j i}-R(\alpha) c_{j i}\right) \\
& +\frac{\delta K}{2}\left(\sum_{k} \omega_{k j}-\sum_{k} \omega_{k i}\right)-K h_{j} \rho_{j}+K h_{i} \rho_{i} .
\end{aligned}
$$

Note that systems (24) can be written in compact form as

$$
\dot{\bar{q}}=-N K \bar{e}+\frac{\delta K}{2} B_{1} \bar{\omega}+K B_{2} \bar{\rho},
$$

where $B_{1}, B_{2}, \bar{q}$ and $\bar{e}$ are defined in (13) and (9). Note from (19) and $\rho_{i}=\Delta_{\rho, i} \cdot \xi_{i}$ that the input vectors $\bar{\rho}^{T}=\left[\rho_{1}^{T}, \cdots, \rho_{N}^{T}\right]$ and $\bar{\omega}$ (which gathers all terms $\omega_{j i}$, arranged in a similar way as $\bar{q}$ in (9)) satisfy $\bar{\omega}=\bar{\Delta}_{q} \bar{\psi}$ and $\bar{\rho}=\bar{\Delta}_{\rho} \bar{\xi}$ respectively, where $\bar{\psi}, \bar{\xi}, \bar{\Delta}_{q}$ and $\bar{\Delta}_{\rho}$ are augmented vectors and block-diagonal matrices properly arranged containing respectively $\psi_{j i}, \xi_{i}, \Delta_{q, j i}$ and $\Delta_{\rho, j i}$.

From the definition of $\xi_{i}$ in (20) and $\psi_{j i}=\dot{q}_{j i}$, note also that $\bar{\xi}$ and $\bar{\psi}$ can be written in compact form as

$$
\bar{\psi}=-N K \bar{e}+\frac{\delta K}{2} B_{1} \bar{\omega}+K B_{2} \bar{\rho}, \quad \bar{\xi}=\delta D \bar{\omega} .
$$

Finally, noting from Lemma 1 that $\left\|\Delta_{q, j i}\right\|_{\infty} \leq 1$, $\left\|\Delta_{\rho, i}\right\|_{\infty} \leq 1$ and defining $\Delta=\operatorname{diag}\left(\bar{\Delta}_{q}, \bar{\Delta}_{\rho}\right)$, the proof can be concluded by defining $\eta^{T}=\left[\bar{\omega}^{T}, \bar{\rho}^{T}\right]$ and $y^{T}=\left[\bar{\psi}^{T}, \bar{\xi}^{T}\right]$.

Notice that the two terms introduced in the rightmost part of (6) have been introduced to remove the delayed terms with $\underline{\tau}$ and $\bar{\tau}$ in (22) by setting $\gamma=1$. Consequently, the proposed control allows to find a delay-free equivalent model for the closed-loop system, as stated 
in Lemma 2. From this equivalence, next sections adapt classical results of robust control theory to obtain sufficient conditions for robust stability and control gain synthesis.

\subsection{Sufficient LMI condition for robust stability}

This section addresses the asymptotic stability analysis with decay-rate performance $\beta$ (hereafter, referred to as $\beta$-stability) of the formation control system (1) with predictor-feedback control $(6)(\gamma=1)$ given a timevarying delay interval $\delta=\bar{\tau}-\underline{\tau}$. Then, a sufficient condition based on LMI for robust $\beta$-stability is obtained by exploiting the equivalent delay-free interconnected model given in Lemma 2, together with the Lyapunov's direct method and small gain theory (see Theorem 2)

Theorem 2 Given a time-varying delay interval $\delta$ and a control gain $K>0$, the formation control system (1)(6) with $\gamma=1$ is robustly $\beta$-stable for any time-varying delays $\underline{\tau} \leq \tau_{j i}(t) \leq \bar{\tau}$ if there exist scalars $\mu>0, \mu_{i}>$ $0, i=1, \cdots, N-1$ and symmetric matrices $S_{i}>0\left(S_{i} \in\right.$ $\left.\mathcal{R}^{2}\right)$ with $i=1, \cdots, \bar{N}$ such that $\Omega<0$, where

$$
\Omega=\left[\begin{array}{ccc}
2 \mu K \mathcal{A}_{s}+2 \mu \beta \cdot I_{\bar{N}} & \mu K \mathcal{B}_{s} L_{\delta} & \mathcal{C}_{s}^{T} L_{K} W_{2} \\
(*) & -W_{1} & L_{\delta} \mathcal{D}_{s}^{T} L_{K} W_{2} \\
(*) & (*) & -W_{2}
\end{array}\right],
$$

$W_{1}=\operatorname{diag}\left(Z, X_{1}\right), \quad W_{2}=\operatorname{diag}\left(Z, X_{2}\right)$,

$Z=\operatorname{diag}\left(S_{1}, \cdots, S_{\bar{N}}\right)$,

$X_{1}=\operatorname{diag}\left(I_{2}, \mu_{1} I_{2}, \cdots, \mu_{N-1} I_{2}\right)$,

$X_{2}=\operatorname{diag}\left(1, \mu_{1}, \cdots, \mu_{N-1}\right)$

and $L_{\delta}, L_{K}, \mathcal{A}_{s}, \mathcal{B}_{s}, \mathcal{C}_{s}, \mathcal{D}_{s}$ are defined in (12).

Proof: Let us consider the following Lyapunov function:

$$
V=\frac{\mu}{N} \sum_{i} \sum_{k} \sum_{j}\left(q_{k j}-R\left(\alpha_{i}\right) c_{k j}\right)^{2},
$$

where $\mu>0$. The time-derivative $\dot{V}$ yields

$$
\dot{V}=\frac{\mu}{N} \sum_{i} \sum_{k} \sum_{j}\left(\frac{\partial V}{\partial q_{k j}} \dot{q}_{k j}+\frac{\partial V}{\partial \alpha_{i}} \dot{\alpha}_{i}\right) .
$$

Noting that $V=\frac{\mu}{N} \sum_{i} J_{i}$, where $J_{i}$ is the cost function $J_{i}=\sum_{k} \sum_{j}\left\|q_{k j}-R\left(\alpha_{i}\right) c_{k j}\right\|^{2}$, we have that $\frac{\partial V}{\partial \alpha_{i}}=$ $0, \forall i=1, \ldots, N$ since $\alpha_{i}=\operatorname{argmin}\left(J_{i}\right)$. Then, (29) yields

$$
\begin{aligned}
& \dot{V}=\mu \sum_{k} \sum_{j}\left(\frac{\partial V}{\partial q_{k j}} \dot{q}_{k j}\right) \\
& =2 \mu \sum_{k} \sum_{j}\left(q_{k j}-R\left(\alpha_{i}\right) c_{k j}\right) \dot{q}_{k j}=2 \mu \bar{e}^{T} \dot{\bar{q}},
\end{aligned}
$$

where $\bar{e}$ and $\bar{q}$ are defined in (9) and (10). From (11), we can replace $\dot{\bar{q}}$ into (30) obtaining

$$
\dot{V}=2 \mu \bar{e}^{T}\left(K \mathcal{A}_{s} \bar{e}+K \mathcal{B}_{s} L_{\delta} \eta\right) .
$$

On the other hand, by virtue of Theorem 1 , the interconnected system (11) is $\beta$-stable if the forward system $M$ is internally $\beta$-stable, and the condition $\left\|\mathcal{T}_{2} \circ M \circ \mathcal{T}_{1}^{-1}\right\|_{\infty} \leq$ 1 is satisfied. In this case, the following inequality is true:

$$
\dot{V}+2 \beta V+y^{T} W_{2} y-\eta^{T} W_{1} \eta<0,
$$

where $W_{1}=\mathcal{T}_{1}^{T} \mathcal{T}_{1}$ and $W_{2}=\mathcal{T}_{2}^{T} \mathcal{T}_{2}$. Replacing $\dot{V}$ from (31) into (32) and taking into account from (11) that $y=$ $L_{K} \mathcal{C}_{s} \bar{e}+L_{K} \mathcal{D}_{s} L_{\delta} \eta$, we deduce that (32) is equivalent to

$$
\left[\begin{array}{ll}
\bar{e}^{T} & \eta^{T}
\end{array}\right] \cdot\left[\begin{array}{ll}
\Omega_{11} & \Omega_{12} \\
\Omega_{12}^{T} & \Omega_{22}
\end{array}\right] \cdot\left[\begin{array}{l}
\bar{e} \\
\eta
\end{array}\right]<0,
$$

where

$$
\begin{aligned}
& \Omega_{11}=2 \mu K \mathcal{A}_{s}+\mathcal{C}_{s}^{T} L_{K} W_{2} L_{K} \mathcal{C}_{s}+2 \mu \beta \cdot I_{\bar{N}}, \\
& \Omega_{12}=\mu K \mathcal{B}_{s} L_{\delta}+\mathcal{C}_{s}^{T} L_{K} W_{2} L_{K} \mathcal{D}_{s} L_{\delta} \\
& \Omega_{22}=-W_{1}+L_{\delta} \mathcal{D}_{s}^{T} L_{K} W_{2} L_{K} \mathcal{D}_{s} L_{\delta} .
\end{aligned}
$$

The above inequality holds $\forall \bar{e}, \eta \neq 0$ if and only if $\bar{\Omega}<0$. Applying Schur Complement, it can be proved that the inequality $\Omega<0$ is true if and only if $\bar{\Omega}<0$, which is a sufficient condition for the robust $\beta$-stability of (11). Finally, from the equivalence stated in Lemma 2 between (11) and the formation control (1) with (6) with $\gamma=1$, the proof is completed.

The corollary given below shows that delay compensation tends to be exact as long as the time-varying delay interval $\delta$ is smaller. Hence, if $\delta$ is sufficiently small, the formation control (6) guarantees the $\beta$-stability by setting $\gamma=1$ and any control gain satisfying $K>\beta / N$.

Corollary 1 The inequality $\Omega<0$ in Theorem 2 is always true for any time-varying delay interval $\delta<\delta^{*}$ with $\delta^{*}>0$ sufficiently small, provided that $K>\beta / N$ in (6) with $\gamma=1$.

Proof: Let $W_{1}=\operatorname{diag}\left(w_{1} I_{2} \bar{N}, w_{2} I_{2 N}\right)$ and $W_{2}=$ $\operatorname{diag}\left(w_{1} I_{2} \bar{N}, w_{2} I_{N}\right)$ in $(27)$, for some positive scalars $w_{1}, w_{2}$. Then, it can be proved that by setting $\delta=0$, there exist sufficiently small values for $w_{1}, w_{2}^{-1}$ such that $\Omega<0$ fulfils for any $K>\beta / N$. Finally, the inequality $\Omega<0$ still holds by continuity for any $\delta<\delta^{*}$ if $\delta^{*}$ is small enough. 


\section{Control synthesis}

The control gain $K$ for the predictor-feedback control (6) $(\gamma=1)$ can be designed in order to maximize the time-varying delay interval $\delta=\bar{\tau}-\underline{\tau}$ for a prescribed decay rate $\beta$ by combining Algorithm 1 and Algorithm 2 (see below): Algorithm 1 finds by dichotomic search the maximum $\delta$ for some $K, \beta$ by means of Theorem 2 , and Algorithm 2 finds by dichotomic search the control gain $K$ that maximizes $\delta$ for some $\beta$ by using Algorithm 1 .

Note that a starting feasible solution is guaranteed for any $K^{*}>\beta / N$ and $\delta^{*}>0$ sufficiently small, in view of Corollary 1. Other parameters involved in Algorithms 1 and 2 are the step increments for $\delta$ and $K\left(\Delta_{\delta}, \Delta_{K}>0\right.$ respectively), the step reduction factors $0<\gamma_{\delta}<1$, $0<\gamma_{K}<1$, and the maximum relative errors $\epsilon_{\delta}(\%)>0$, $\epsilon_{K}(\%)>0$.

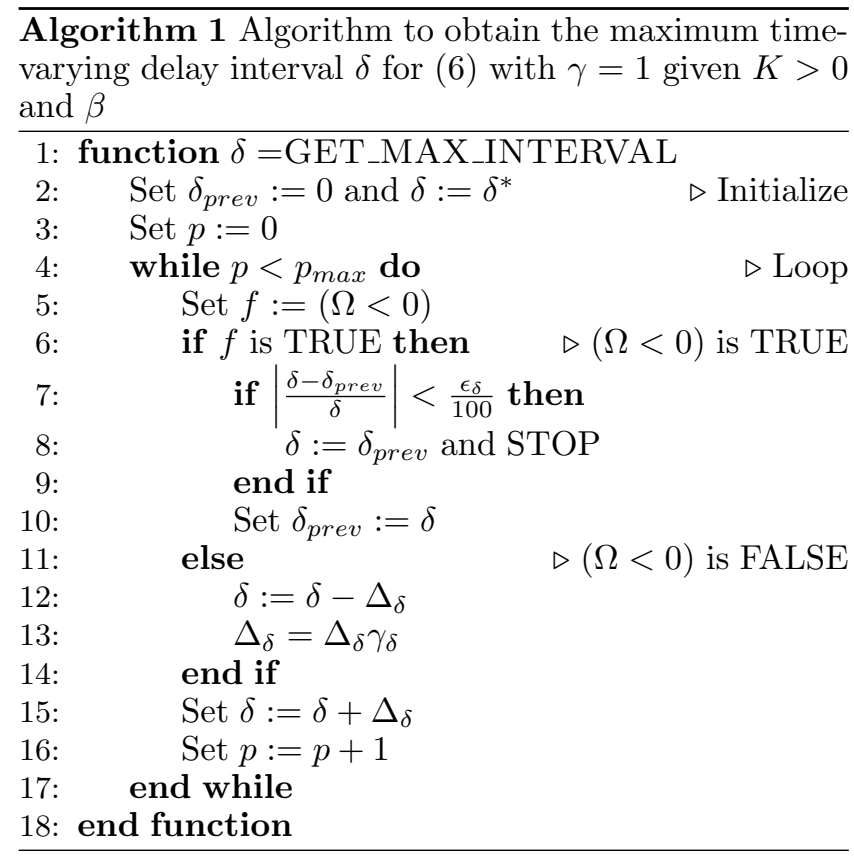

\section{Simulations}

Two simulation examples are provided in this section. The first one illustrates the trade-offs between the maximum time-varying delay interval, the decay rate and number of agents. The second example confirms the effectiveness of the control gain synthesis $K$ given in Algorithm 2 by comparing the achieved performance with other values for $K$. Both examples consider different target formations and show the superiority of the predictorfeedback with respect to no delay compensation [9].

\subsection{Example 1}

Consider a circular target formation of radius $6 \mathrm{~m}$ and a certain number of agents $N$, where the agent's positions are equally distributed around the circle (see Fig.

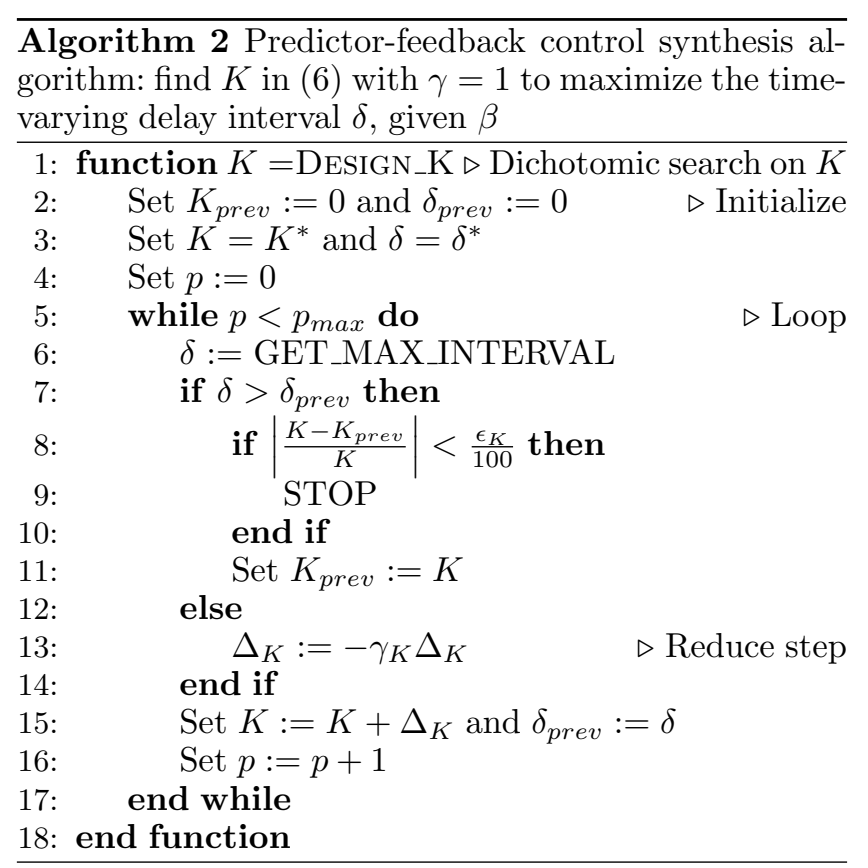

1 with $N=4,6,8)$. By applying Algorithm 2, we obtain the control gain $K$ with the proposed predictor-feedback (6) with $\gamma=1$ that maximizes the time-varying delay interval $\delta$ for different number of agents $N$ and different decay rates $\beta$.

The obtained results are depicted in Fig. 2, where it can be appreciated that the maximum achievable timevarying delay interval $\delta$ diminishes as long as the decay rate $\beta$ increases. This fact reveals a trade-off between robust performance and convergence speed in the predictor-feedback control design. A similar trade-off can be appreciated in the compromise between robust performance and number of agents, since the maximum achievable time-varying delay interval $\delta$ diminishes when the number of agents $N$ grows. Note also that smaller values of $H$ defined in Assumption 1 give more pessimistic estimations of $\delta$ (as could be expected from the bounding techniques used to linearize the control synthesis problem), but the designed control gain $K$ that maximizes $\delta$ is hardly influenced by $H$ in all cases (see left side in Fig. 2 and Fig. 3). The following values have been chosen for Algorithm 2: $\Delta_{K}=10^{-3}, \gamma_{K}=0.5$, $\Delta_{\delta}=1 s, \gamma_{\delta}=0.5$ and maximum relative errors for $K$ and $\delta: \epsilon_{K}=1 \%, \epsilon_{\delta}=1 \%$. As a result, the total number of iterations between Algorithm 1 and Algorithm 2 ranges between $200-300$ considering starting values $K^{*}=\beta / N+10^{-6}$ and $\delta^{*}=10^{-6}$. Also, the number of iterations in Algorithm 2 ranges between $15-25$.

Let $N=4,6,8$. Taking into account the condition $K>$ $\beta / N$, a minimum gain $K=2.5 \cdot 10^{-3}, K=1.7 \cdot 10^{-3}$ and $K=1.3 \cdot 10^{-3}$ are necessary for each case in the absence of delays. Nevertheless, all three systems become unstable for delays larger than $150 \mathrm{~s}$ using [9] with any control gain $K$ larger than those values. Hence, a settling 


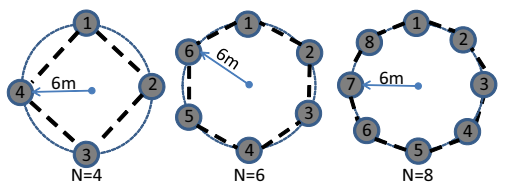

Fig. 1. Different target formations for the three cases under analysis with number of agents: $\mathrm{N}=4, \mathrm{~N}=6$ and $\mathrm{N}=8$ respectively.
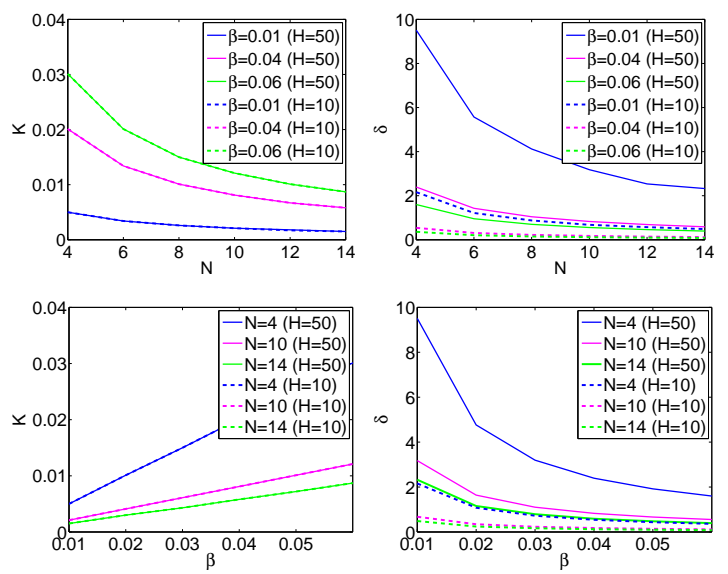

Fig. 2. Designed control gain $K$ (left-column) and the maximum delay interval $\delta$ (right column) for different number of agents $N$ (upper row) and decay rate $\beta$ (lower row).

time of $300 \mathrm{~s}$ is not possible without delay compensation.

Now, let us proceed by simulation to show if the target formation can be reached in approximately 300s $(\beta=0.01)$ with the designed predictor-feedback control under time-varying delays $\tau_{j i}(t) \in(120 s, 180 s), \forall i, j$ with time-varying delay interval $\tau=60 \mathrm{~s}$ and average delay $150 \mathrm{~s}$. In view of the results given in the second row of Fig. 4, the target formation is achieved for $N=4$ (left column), $N=6$ (middle column) and $N=8$ (right column), whereas the system is unstable without delay compensation in all three cases. The time evolution of the normalized cost function $J(t) / J(0)$ with $J=\sum_{i} \sum_{k} \sum_{j}\left\|q_{k j}-R\left(\alpha_{i}\right) c_{k j}\right\|^{2}$ is depicted in Fig. 5. The initial agents' positions are $q_{i}(t=0)=D_{\max }\left(\Theta_{q}-\right.$ $0.5\left[\begin{array}{ll}1 & 1\end{array}\right]^{T}$ ) (depicted by "o" symbols in Fig. 4), where $\Theta_{q}$ provides a $2 \times 1$ pseudorandom value with a uniform distribution on $(0,1)$, and $D_{\max }=15 \mathrm{~m}$ stands for the maximum distance between agents.

\subsection{Example 2}

Consider the multi-agent system (1) with $N=12$ agents, and the prescribed target formation depicted in the left side of Fig. 6. For control synthesis, we establish a maximum settling time of $300 \mathrm{~s}$, which approximately corresponds to $\beta=0.01$. The control gain $K$ for (6) with $\gamma=1$ is designed by Algorithm 2 to maximize $\delta$, obtaining $K=1.7 \cdot 10^{-3}$ (see Fig. 6 , middle side). Now,
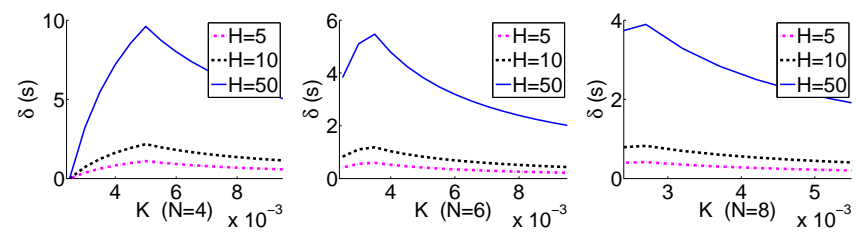

Fig. 3. Maximum delay interval $\delta$ vs controller gain $K$ (obtained by Algorithm 1 using different values of $H$ and $\beta=0.01$ ) for the three target formations given in Fig. 1: From left to right: $N=4,6$ and 8 agents respectively.
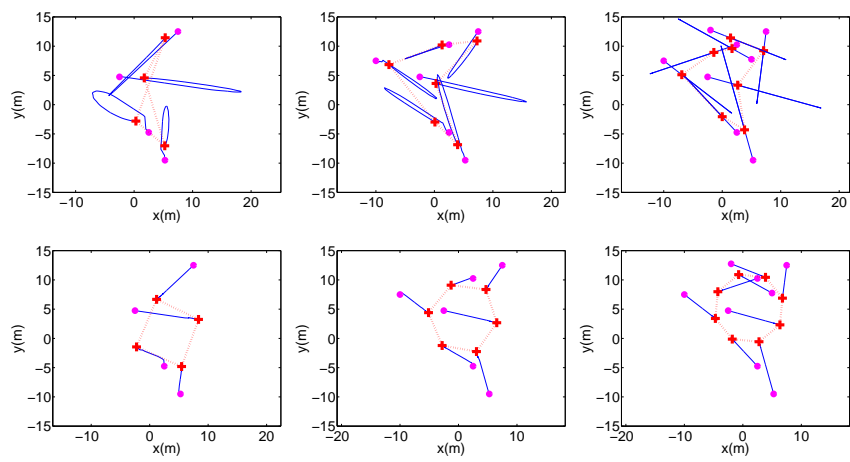

Fig. 4. Trajectories followed by each agent (Example 1) for each target formation $N=4,6$ and 8 under time-varying delays $120 \mathrm{~s} \leq \tau_{j i}(t) \leq 180 \mathrm{~s}$ : Left $\left(\mathrm{K}=5.0 \cdot 10^{-3}\right)$, Middle $\left(\mathrm{K}=3.3 \cdot 10^{-3}\right)$ and Right $\left(2.6 \cdot 10^{-3}\right)$. First row: no delay compensation, Second row: proposed predictor-feedback control.
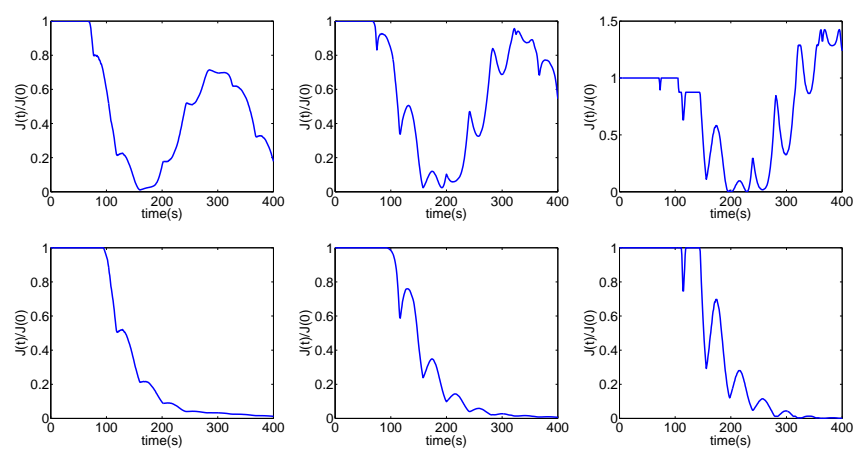

Fig. 5. Normalized cost function $J(t) / J(0)$ for Example 1 , corresponding to each target formation $N=4,6$ and 8 under time-varying delays $120 \mathrm{~s} \leq \tau_{j i}(t) \leq 180 \mathrm{~s}$ : Left $\left(\mathrm{K}=5.0 \cdot 10^{-3}\right)$, Middle $\left(\mathrm{K}=3.3 \cdot 10^{-3}\right)$ and Right $\left(2.6 \cdot 10^{-3}\right)$. First row: no delay compensation, Second row: proposed predictor-feedback control.

consider a maximum settling time $30 \mathrm{~s}$ (ten times faster, $\beta=0.1$ ). In this case, we obtain $K=1.6 \cdot 10^{-2}$ (see Fig. 6 , right side). Note that the maximum time-varying delay interval $\delta$ is smaller when $\beta=0.1$, which confirms again that faster convergence is at the expense of loss of robustness against time-varying delays.

Comparative simulation results between predictorfeedback formation control $(\gamma=1$ in (6)) and nonpredictor case $(\gamma=0)$ are given below for differ- 

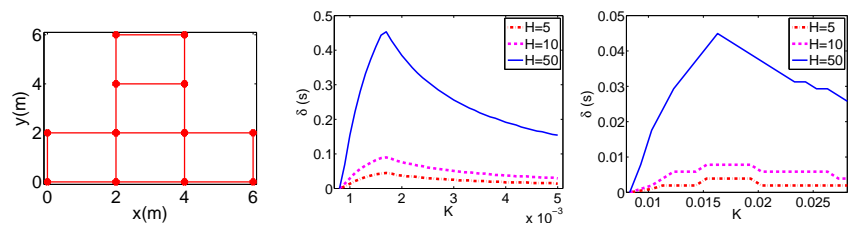

Fig. 6. Left-side: Target formation to be reached (Example 2 ). Middle and right side: maximum delay interval $\delta$ vs controller gain $K$ with $\beta=0.01$ and $\beta=0.1$, respectively

ent control gains considering $\beta=0.01$. Let the initial position vectors of each agent described by $q_{i}(t=0)=D_{\max }\left(\Theta_{q}-0.5\left[\begin{array}{ll}1 & 1\end{array}\right]^{T}\right)$ where $\Theta_{q}$ provides a $2 \times 1$ pseudorandom value with a uniform distribution on $(0,1)$ and $D_{\max }=15 \mathrm{~m}$ stands for the maximum distance between agents. We consider the same randomly pre-generated time-varying delay patterns $\tau_{j i}(t) \in(60 s, 90 s)$ for a fair comparison.

The results given in the first row of Fig. 7, Fig. 8, Fig. 9 and Fig. 10 correspond to the non-predictor formation control (6) with $\gamma=0$ (no delay compensation) for three different control gains: $K=0.4 \cdot 10^{-3}$ (left column), $K=1.7 \cdot 10^{-3}$ (middle column) and $K=10 \cdot 10^{-3}$ (right column). The first row of Fig. 7 depicts the initial agents' positions ("o" symbols), the trajectories followed by each agent (solid line) and the final agents' position ("+" symbols) after $300 s$ of time simulation using different K. Fig. 8 and Fig. 9 (first-row) depict their corresponding velocity norms and estimated rotation angles respectively. By choosing $K=0.4 \cdot 10^{-3}$ the prescribed target formation is not achieved after $300 \mathrm{~s}$ (see first column in the first row of Fig. 10), but choosing greater values of $K$ lead the system to instability (see the second and third columns in the first row of Fig. 10). Hence, the required settling time of $300 \mathrm{~s}$ cannot be achieved for any control gain $K$ without delay compensation.

Nevertheless, it can be appreciated that the system performance clearly improves by introducing the predictorfeedback by setting $\gamma=1$ in (6), specially for higher values of $K$ (see comparison between the first and the second row in Fig. 7, Fig. 8, Fig. 9 and Fig. 10, middle and right columns). Moreover, the control gain $K=1.7 \cdot 10^{-3}$ designed by Algorithm 2 gives the best performance in comparison with the other choices for $K$. Indeed, note that smaller values than $1.7 \cdot 10^{-3}$ for $K$ gives slow convergence, and greater values for $K$ exhibit visible perturbations in the agents' trajectories due to higher sensitivity against time-varying delay mismatches. This comparison corroborates the effectiveness of the proposed control gain synthesis given in Algorithm 2.

\section{Conclusions}

In this paper, predictor-feedback delay compensation has been proposed to enhance robust performance in
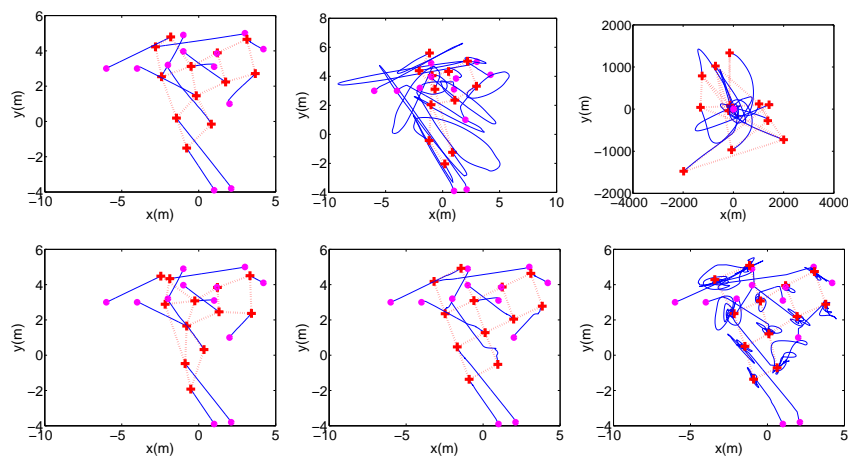

Fig. 7. Trajectories followed by each agent (Example 2) using different $K$ with $60 s \leq \tau_{j i}(t) \leq 90 \mathrm{~s}$ : Left $\left(K=0.4 \cdot 10^{-3}\right)$, Middle $\left(K=1.7 \cdot 10^{-3}\right)$ and Right $\left(K=10 \cdot 10^{-3}\right)$. First row: no delay compensation. Second row: proposed predictor-feedback control.
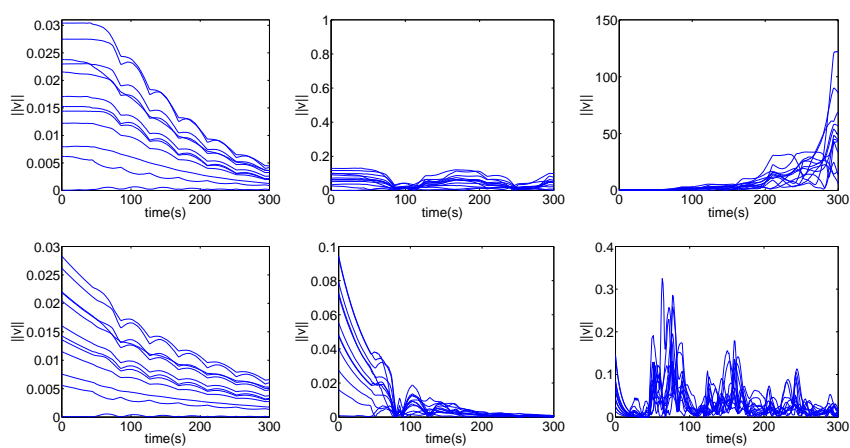

Fig. 8. Velocity norm $\left\|v_{i}\right\|$ of each agent (Example 2) using different $K$ with $60 s \leq \tau_{j i}(t) \leq 90 s$ : Left $\left(K=0.4 \cdot 10^{-3}\right)$, Middle $\left(K=1.7 \cdot 10^{-3}\right)$ and Right $\left(K=10 \cdot 10^{-3}\right)$. First row: no delay compensation. Second row: proposed predictor-feedback control.
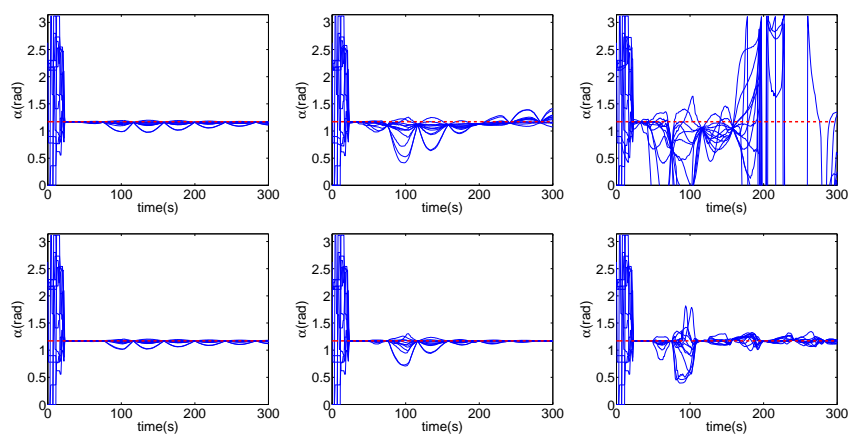

Fig. 9. Rotation angle $\alpha_{i}$ computed by each agent (Example 2) using different $K$ with $60 s \leq \tau_{j i}(t) \leq 90 \mathrm{~s}$ : Left $\left(K=0.4 \cdot 10^{-3}\right)$, Middle $\left(K=1.7 \cdot 10^{-3}\right)$ and Right $\left(K=10 \cdot 10^{-3}\right)$. First row: no delay compensation. Second row: proposed predictor-feedback control..

coordinate-free formation control under unknown timevarying communication delays. By introducing two delayed terms in the predictor-feedback control, the lower and upper delay bounds are compensated and hence eliminated from the closed-loop system model. More- 

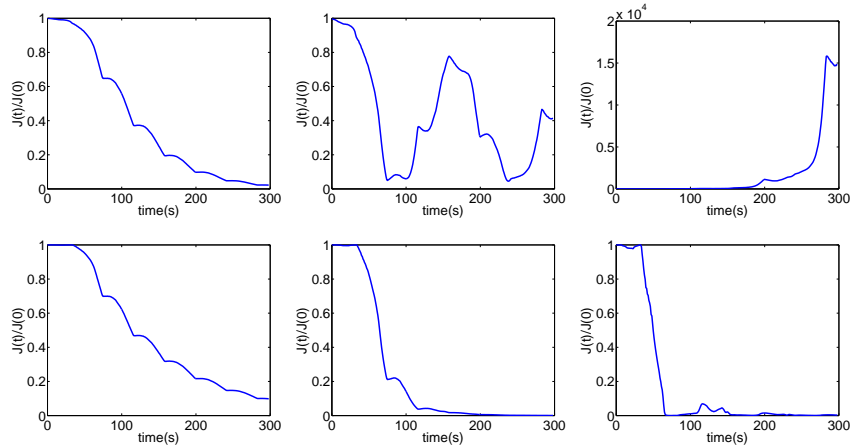

Fig. 10. Normalized cost function $J(t) / J(0)$ for Example 2 using different $K$ with $60 s \leq \tau_{j i}(t) \leq 90 \mathrm{~s}$ : Left $\left(K=0.4 \cdot 10^{-3}\right)$, Middle $\left(K=1.7 \cdot 10^{-3}\right)$ and Right $\left(K=10 \cdot 10^{-3}\right)$. First row: no delay compensation. Second row: proposed predictor-feedback control.

over, larger time-varying delay intervals are allowed by designing the control gain through efficient algorithms based on LMI. Finally, simulation results have illustrated the effectiveness of the control synthesis in the aim of improving the existing trade-offs between robustness against time-varying delay uncertainties, convergence speed and number of agents.

\section{References}

[1] X. Dong, B. Yu, Z. Shi, Y. Zhong, Time-varying formation control for unmanned aerial vehicles: Theories and applications, IEEE Transactions on Control Systems Technology 23 (1) (2015) 340-348.

[2] J. L. Baxter, E. Burke, J. M. Garibaldi, M. Norman, Multirobot search and rescue: A potential field based approach, in: Autonomous robots and agents, Springer, 2007, pp. 9-16.

[3] H. Bai, J. T. Wen, Cooperative load transport: A formationcontrol perspective, IEEE Transactions on Robotics 26 (4) (2010) 742-750.

[4] K.-K. Oh, M.-C. Park, H.-S. Ahn, A survey of multi-agent formation control, Automatica 53 (2015) 424-440.

[5] K.-K. Oh, H.-S. Ahn, Formation control of mobile agents based on inter-agent distance dynamics, Automatica 47 (10) (2011) 2306-2312.

[6] M. M. Zavlanos, G. J. Pappas, Distributed formation control with permutation symmetries, in: 46th IEEE Conference on Decision and Control, 2007, pp. 2894-2899.

[7] D. V. Dimarogonas, K. J. Kyriakopoulos, A connection between formation infeasibility and velocity alignment in kinematic multi-agent systems, Automatica 44 (10) (2008) $2648-2654$

[8] K.-K. Oh, H.-S. Ahn, Formation control and network localization via orientation alignment, IEEE Transactions on Automatic Control 59 (2) (2014) 540-545.

[9] M. Aranda, G. López-Nicolás, C. Sagüés, M. M. Zavlanos, Coordinate-free formation stabilization based on relative position measurements, Automatica 57 (2015) 11-20.

[10] S.-I. Niculescu, Delay effects on stability: a robust control approach, Vol. 269, Springer Science \& Business Media, 2001.

[11] K. Gu, J. Chen, V. L. Kharitonov, Stability of time-delay systems, Springer Science \& Business Media, 2003.
[12] B. Zhou, Z. Lin, Consensus of high-order multi-agent systems with large input and communication delays, Automatica 50 (2) (2014) 452-464.

[13] H. Liu, H. R. Karimi, S. Du, W. Xia, C. Zhong, Leaderfollowing consensus of discrete-time multiagent systems with time-varying delay based on large delay theory, Information Sciences 417 (2017) $236-246$.

[14] W. Li, Z. Chen, Z. Liu, Leader-following formation control for second-order multiagent systems with time-varying delay and nonlinear dynamics, Nonlinear Dynamics 72 (4) (2013) 803-812.

[15] L. Han, X. Dong, Q. Li, Z. Ren, Formation-containment control for second-order multi-agent systems with timevarying delays, Neurocomputing 218 (2016) 439-447.

[16] T. Li, Z. Li, H. Zhang, S. Fei, Formation tracking control of second-order multi-agent systems with time-varying delay, Journal of Dynamic Systems, Measurement, and Control 140 (11) (2018).

[17] J. E. Normey-Rico, E. F. Camacho, Control of dead-time processes, Time (minutes) Time (minutes).

[18] A. González, A. Sala, R. Sanchis, LK stability analysis of predictor-based controllers for discrete-time systems with time-varying actuator delay, Systems \& Control Letters 62 (9) (2013) 764-769.

[19] M. Krstic, Lyapunov stability of linear predictor feedback for time-varying input delay, IEEE Transactions on Automatic Control 55 (2) (2010) 554-559.

[20] N. Bekiaris-Liberis, M. Krstic, predictor-feedback stabilization of multi-input nonlinear systems, IEEE Transactions on Automatic Control 62 (2) (2017) 516-531.

[21] A. González, M. Aranda, G. López-Nicolás, C. Sagüés, Time delay compensation based on Smith predictor in multiagent formation control, IFAC-PapersOnLine 50 (1) (2017) 1164511651.

[22] P. Gahinet, A. Nemirovski, A. Laub, M. Chilali, LMI control toolbox, The MathWorks Inc. 1995.

[23] Y. Labit, D. Peaucelle, D. Henrion, SeDuMi interface 1.02: a tool for solving LMI problems with SeDuMi, in: IEEE International Symposium on Computer Aided Control System Design, 2002, pp. 272-277.

[24] A. González, M. Aranda, G. López-Nicolás, C. Sagüés, Robust stability analysis of formation control in local frames under time-varying delays and actuator faults, Journal of the Franklin Institute 356 (2) (2019) 1131-1153.

[25] K. Zhou, J. C. Doyle, Essentials of robust control, Vol. 104, Prentice Hall, Upper Saddle River, NJ, 1998.

[26] L. Zhao, H. Gao, H. R. Karimi, Robust stability and stabilization of uncertain T-S fuzzy systems with timevarying delay: an input-output approach, IEEE Transactions on Fuzzy Systems 21 (5) (2013) 883-897.

[27] Z. Artstein, Linear systems with delayed controls: a reduction, IEEE Transactions on Automatic control 27 (4) (1982) 869-879.

[28] D. V. Dimarogonas, K. J. Kyriakopoulos, On the rendezvous problem for multiple nonholonomic agents, IEEE Transactions on Automatic Control 52 (5) (2007) 916-922.

[29] M. Jafarian, Robust consensus of unicycles using ternary and hybrid controllers, International Journal of Robust and Nonlinear Control 27 (17) (2017) 4013-4034. 